\title{
Mercadeo de experiencias en odontología por medio de la evaluación de respuesta fisiológica y emocional en individuos expuestos al eugenol
}

David Juliao Esparragoza*

Domingo Martinez Diaz ${ }^{* *}$

Ingrid Insignares ${ }^{\star \star *}$

Fecha de recibido: 19 de agosto de 2019 Fecha de aprobado: 12 de diciembre de 2019

Para citar este artículo: Juliao Esparragoza, D., Martinez Díaz, D., \& Insignares, I. (2020). Mercadeo de experiencias en odontología por medio de la evaluación de respuesta fisiológica y emocional en individuos expuestos al eugenol. Revista Universidad \& Empresa, 22(39), 1-23. https://doi.org/10.12804/revistas.urosario.edu.co/

empresa/a.8101

* Economista. Magíster en administración de empresas. Profesor asistente, Universidad del Norte, Barranquilla, Colombia. Correo electrónico: djuliae@uninorte.edu.co

** Estadístico. Doctor en psicología. Profesor asistente, Universidad del Norte, Barranquilla, Colombia. Correo electrónico: domimart@uninorte.edu.co

*** Odontóloga. Estudiante de la Maestría en Mercadeo, Universidad del Norte, Barranquilla, Colombia. Correo electrónico: ingridinsignares@hotmail.com 


\title{
Resumen
}

Esta propuesta de investigación utiliza los análisis de entrevistas a profundidad y la biometría para identificar cómo impactan los estímulos olfativos relacionados con la consulta odontológica en la emoción de los sujetos investigados. Primero se realiza una explicación sobre las características inherentes a la experiencia de mercadeo y cómo esta puede generar la cascada de sensaciones, percepciones y emociones buscadas en bienes y servicios con características hedónicas de consumo. Hoy el consumo de servicios odontológicos tiene varios frentes comerciales. En las diferentes especialidades se encuentra la estética, que normalmente, se busca como consumo placentero. Así mismo, la variable estrés, comúnmente estudiada en este servicio, genera animadversión a la consulta y se asocia a emociones negativas. El estudio identifica al estímulo olfativo y específicamente su relación con las emociones como una de las variables que contribuye al estrés. La investigación permitió concluir que el estímulo olfativo a eugenol tiene impactos identificables de estrés y emociones negativas asociadas en pacientes que tuvieron experiencias negativas en odontología asociadas a este olor. Se demuestra que el efecto de condicionamiento clásico (Till \& Priluck, 2000) por olor es claro y se identifican opciones de mejora para generar ambientes que generen experiencias placenteras en el mercado meta.

Palabras clave: marketing de experiencias; evaluación emocional; biometría.

\section{Marketing of Experiences in Dentistry through the Evaluation of Physiological and Emotional Response in Individuals Exposed to Eugenol}

\begin{abstract}
This research uses biometrics to identify how olfactory stimuli related to dental consultation affect the emotion of the subjects investigated. First, an explanation is given about the characteristics inherent to experience marketing and how it can generate a cascade of sensations, perceptions, and emotions normally sought in goods and services with hedonic consumption characteristics. Today the consumption of dental services has several commercial fronts. In the different specialties aesthetics is part of them, which is normally sought as pleasant consumption. Likewise, the stress variable, commonly studied in this service, generates animosity to the consultation and is associated with negative emotions. The study identifies one of the variables that contributes to stress in dentistry, such as the olfactory stimulus, and specifically its relationship with emotions. The research allows concluding, by means of a field research, that the olfative stimulus to eugenol has identifiable impacts on stress and is associated with negative emotions in patients who have had negative experiences in dentistry. It is shown that the effect of classical conditioning (Till \& Priluck, 2000) by smell is clear and improvement options are identified to generate environments that generate pleasurable experiences in the target market.
\end{abstract}

Keywords: Experiential marketing; emotional values; biometrics.

\section{Marketing de experiências em odontologia por meio da avaliação de resposta fisiológica e emocional em indivíduos expostos ao eugenol}

\section{Resumo}

Esta proposta de pesquisa utiliza as análises de entrevistas a profundidade e a biometria para identificar como impactam os estímulos olfativos relacionados com a consulta odontológica na emoção dos sujeitos pesquisados. Primeiro se realiza uma explicação sobre as características inerentes à experiência em marketing e como estas podem gerar a cascata de sensações, percepções e emoções normalmente buscada em bens e serviços com características hedónicas de consumo. Hoje o consumo de serviços odontológicos tem vários campos comerciais. Nas diferentes especialidades se encontram as estéticas, que normalmente, buscam-se como consumo prazenteiro. Igualmente, a variável estresse, comumente estudada neste serviço, gera animadversão à consulta e associa-se a emoções negativas. O estudo identifica uma das variáveis que contribui ao estresse em odontologia como é o estímulo olfativo e especificamente sua relação 
com as emoções. Conclui-se mediante a pesquisa de campo que o estímulo olfativo a eugenol tem impactos identificáveis de estresse e emoções negativas associadas em pacientes que tiveram experiências negativas em Odontologia associadas a este cheiro. Demostra-se que o efeito de condicionamento clássico (Till \& Priluck, 2000) por cheiro é claro e se identificam opções de melhora para gerar ambientes que gerem experiências prazenteiras no mercado alvo.

Palavras-chave: marketing de experiências; avaliação emocional; biometria.

\section{Introducción}

El mercadeo de experiencias ha sido estudiado ampliamente (Holbrook \& Hirshman, 1982; Schmitt, 1999; Pine \& Gilmore, 1999; Frow \& Payne, 2007; Gentille, Spiller \& Noci, 2007). Su enfoque requiere de la asistencia y entendimiento de los estímulos en las percepciones para crear asociaciones positivas que refuercen atributos emocionales de marca (Mukiira, Musau \& Munyao, 2017). El cual es ampliamente aplicado tanto en el mercado de bienes como en el de servicios, por lo que la odontología no debe ser ajena a este.

La percepción de estímulos tiene estrechas relaciones con fenómenos vividos (experiencia), ya que esta crea aprendizajes y correlatos emocionales. Este fenómeno estudiado por el condicionamiento clásico puede ponerse en práctica en el entendimiento y construcción de ambientes de consulta en odontología donde los estímulos más recordados en experiencias traumáticas son el estímulo sonoro y el olfativo (Carballo, 1997). Se entiende a partir de narrativas y análisis biométricos que la sensación producida en grupos con experiencias traumáticas en odontología responde negativamente a los estímulos mencionados.

$\mathrm{Al}$ identificar una de las variables que influye negativamente en la percepción del sujeto investigado se puede inferir que la modificación en el estímulo podría generar sensaciones nuevas en el ambiente y realizar un reacondicionamiento o reaprendizaje en el mismo. Quedan investigaciones pendientes a partir de esta que podrían diferenciar relaciones entre grupos de manera estadística a partir de Anovas u otras técnicas o modelos de análisis factorial con experimentos más robustos. 


\section{Análisis teórico}

El uso del mercadeo de experiencias cobija una amplia gama de aplicaciones en productos y servicios con implicaciones positivas en términos de lealtad y satisfacción del consumidor (Ming-Shing, Huey-Der \& Ming-Fen, 2010).

El término mercadeo de experiencia fue acuñado seminalmente por los trabajos de Pine y Gilmore a principios de los ochenta. Su enfoque estriba en la visión del cliente de forma holística entendiendo el impacto de sus emociones y la vinculación de estas al proceso de toma de decisiones (Hill, 2017). Las principales características que lo definen frente al mercadeo tradicional se exponen en la tabla 1.

Tabla 1. Diferencias entre el mercadeo tradicional y el mercadeo de experiencias

\begin{tabular}{ll}
\hline \multicolumn{1}{c}{ Mercadeo tradicional } & \multicolumn{1}{c}{ Mercadeo de experiencias } \\
\hline $\begin{array}{l}\text { La elección de compra está en términos de atributos y } \\
\text { beneficios funcionales de productos y servicios. }\end{array}$ & $\begin{array}{l}\text { Las experiencias generan un anclaje en las decisiones de } \\
\text { compra. }\end{array}$ \\
\hline $\begin{array}{l}\text { Los clientes son sujetos racionales en el proceso de } \\
\text { toma de decisiones siempre buscando maximizar su } \\
\text { relación de valor. }\end{array}$ & $\begin{array}{l}\text { Los clientes son esencialmente seres emocionales, cuyas } \\
\text { decisiones pueden afectarse por medio de estímulos } \\
\text { sensoriales, desean ser estimulados mediante experien- } \\
\text { cias positivas. }\end{array}$ \\
\hline $\begin{array}{l}\text { El consumo está circunscrito a la categoría y competen- } \\
\text { cia del producto. }\end{array}$ & $\begin{array}{l}\text { El consumo se define de forma multidimensional, donde } \\
\text { los aportes sociales, culturas y de experiencia juegan un } \\
\text { papel preponderante en las elecciones. }\end{array}$ \\
\hline $\begin{array}{l}\text { Métodos de investigación clásicos donde las variables } \\
\text { dependientes a nivel causal son normalmente indicado- } \\
\text { res robustos como las ventas. }\end{array}$ & $\begin{array}{l}\text { Trata de usar métodos eclécticos y busca dimensionar, } \\
\text { caracterizar o identificar estímulos que conlleven a emo- } \\
\text { ciones o experiencias que ayuden a agregar valor en el } \\
\text { proceso de consumo. }\end{array}$ \\
\hline
\end{tabular}

Fuente: adaptado de Moral y Fernández (2012, p. 239).

El mercado de la salud dental ofrece un nicho propicio para investigar los mejores métodos de acercamiento entre el paciente y el profesional (Morales-García, Higinio \& Priego-Álvarez, 2008). El entendimiento de la mediación de estímulos en la experiencia del paciente puede servir para mejorar sus reacciones en la consulta y evitar respuestas no deseadas con impactos financieros para el profesional y la salud del paciente. ${ }^{1}$ El estudio caracteriza el proceso de

1 Abandono del tratamiento, donde una de las causas probables es la aprehensión o estrés psicológico derivado de experiencias traumáticas previas. Otras causas probables frecuentes estriban en el tiempo del tratamiento y su costo. 
reacción fisiológica y emocional de un sujeto expuesto al eugenol. ${ }^{2}$ Se entiende, de acuerdo al condicionamiento clásico (Rozo \& Pérez, 2015), que si las experiencias odontológicas previas han sido negativas, la exposición a este tipo de sustancias va a disparar una cascada de procesos que, en algunos casos, se traducirá como una señal de peligro al pasar por el sistema límbico al que este normalmente responderá como una amenaza, generando cambios somáticos como el aumento de la presión arterial, frecuencia cardiaca y dilatación pupilar, entre otros. Por medio de este estudio buscamos hacer una asociación entre los estímulos olfativos percibidos en el consultorio dental y la percepción por parte del paciente.

Los tratamientos odontológicos son normalmente considerados un servicio necesario no deseado. Con frecuencia los pacientes advierten experiencias negativas por el paso del consultorio odontológico que afectan la praxis del profesional. Son amplias las aproximaciones entre ciencias de la conducta, especialmente la psicología, y la práctica odontológica (Kleinknecht et al., 1976, citado por Fernández \& Gil, 1994). Algunas de las consecuencias no deseadas de una mala experiencia en el odontólogo, de acuerdo con Caycedo et al. (2013), son

- Miedo desproporcionado y persistente a la consulta odontológica

- Ataque de pánico

- Interferencia o interrupción de tratamiento

Estas respuestas o reacciones no deseadas conducen normalmente a la perpetuación de conductas en detrimento de la salud del paciente y de la práctica odontológica misma.

Se entiende que los niveles de ansiedad son activados mediante estímulos que normalmente son asociados a experiencias previas que condicionan el aprendizaje (Lizardi \& Jiménez, 2012). Así, el primer paso en la experiencia es conducido por la sensación y percepción de estímulos. Este es un tema ampliamente estudiado y está asociado con la manera en la que el cerebro de un organismo interpreta los estímulos sensoriales que recibe a través de los sentidos para formar una idea de la realidad que lo rodea (Goldstein, 2005). "El cerebro procesa estos estímulos, los organiza y almacena según su experiencia

2 Olor característico de la resina para obturar cavidades dentales, que en algunos pacientes genera algún tipo de aprehensión. 
previa, utilidad y características como pensamientos y sentimientos de una manera lógica o significativa" (Goldstein, 2005 p. 36).

La percepción, de acuerdo con Goldstein (2005), se divide en cinco pasos: estímulo, electricidad, experiencia, acción y conocimiento.

El estímulo se refiere a lo que hay en el entorno, que es capaz de producir una alteración en el estado basal $^{3}$ de los receptores. El estímulo al ser recibido se transforma en electricidad en el mismo receptor. A este proceso se le llama transducción. De esta manera, el estímulo viaja a través de los nervios y las neuronas (trasmisión) hasta llegar al cerebro, una vez el estímulo llega al cerebro en forma de estímulos eléctricos, se da el procesamiento del estímulo y su percepción (procesamiento).

La experiencia se refiere a la capacidad de reconocer el estímulo y hacerlo consciente. La acción se refiere a la posición que tomamos frente a este. La percepción entonces es la concientización de un estímulo. Se logra cuando este llega al cerebro y es reconocido, categorizado, asociado a un significado y archivado, para convertirse en una experiencia previa. Goldstein (2005) resume el proceso perceptual como una serie de pasos que comienzan con un estímulo ambiental y terminan con la percepción, el reconocimiento, y la acción.

El conocimiento contextualiza el estímulo en base a las experiencias anteriores que hayamos tenido con este, es decir, aplica el saber frente al estímulo. El conocimiento puede afectar la percepción del estímulo, ya que tiene asociado un proceso de aprendizaje inherente: una vez reconocido un estímulo, será más fácil distinguirlo en una próxima oportunidad.

El estímulo del cual queremos identificar las percepciones y medir los impactos fisiológicos que produce se deriva del olfato cuyas estructuras hacen parte del sistema límbico, asociado a procesos de aprendizaje, reflejos básicos e impulsos primarios (instinto de supervivencia y conservación ante agresiones), procesos de castigo y recompensa y ciclos de sueño. A este nivel se genera la decodificación de la información y se genera

3 Estado basal: conocido como el estado de reposo donde la célula que tiene dicho receptor, en este caso, la neurona no tiene ningún estímulo que la altere. 
una respuesta con base en la experiencia previamente adquirida de las exposiciones con determinado tipo de estímulo olfativo.

El sistema límbico en los animales se reconoce como la primera estructura central asociada a la olfacción y la regulación de emociones y conducta, presumiblemente es la razón por la cual la percepción de olores en animales cumple una función de excitante primario del impulso sexual, por medio de la percepción de feromonas en el proceso de reproducción. El olfato tiene un gran componente emocional hasta tal punto que es el único sentido capaz de evocar vívidamente un recuerdo a través del tiempo, gracias a su núcleo asociado al sistema límbico.

De acuerdo con Childers y Heckler (1987 citados por Avendaño, Paz \& Rueda, 2015), son muchos los estudios que han tratado de incorporar la percepción sensorial en el comportamiento del cliente. Con el desarrollo del mercadeo de experiencias (Pine \& Gilmore, 1998 citados por Moral \& Fernández, 2012) y la incorporación de tecnologías biométricas se abren nuevos espacios para la discusión de fenómenos que antes eran validados solo con recolecciones de datos indirectos y variables de estado mental. ${ }^{4}$

La odontología, como cualquier servicio, podría ofrecer mejores experiencias que evoquen recuerdos positivos, una herramienta para esto es la administración de sensaciones en el consultorio modificando reacciones de luz, temperatura, sonido y aromas (Brakus, Schmitt \& Zarantonello, 2009).

Dado que lo que se requiere es ver la relación entre estímulos y emociones es necesario referirse a estas últimas en términos de su definición, tipologías e impacto en el modelo de toma de decisiones.

Las emociones son tendencias de respuesta con un gran valor adaptativo, que tienen evidentes manifestaciones a nivel fisiológico, en la expresión facial, la experiencia subjetiva, el procesamiento de la información, etc., que son intensas pero breves en el tiempo y que surgen ante la evaluación de algún acontecimiento o antecedente (Vecina-Jiménez, 2006, p.10).

4 Variables difícilmente verificables, corresponden a la respuesta que se hace evidente cuando la persona responde, por ejemplo: ¿El aroma para usted fue agradable o desagradable? La respuesta proviene de una percepción en la cual el investigador confía. 
En la literatura, son muchas las tipologías de las emociones. Existe un debate sobre las diferencias entre estados afectivos (Dienner, 1999); sin embargo, hay convergencia teórica en relación con las emociones básicas cuya base fisiológica es innata. Pertenecen a estas emociones el miedo, la rabia, la alegría y la tristeza (Kemper, 1987). Estas tienen carácter y función adaptativa en términos de evocar energía para adaptarse a la amenaza o ralentizar al organismo como medida adaptativa de protección y cuidado (Páez et al., 1997). El estudio de estas emociones ha sido validado transculturalmente y algunos investigadores encontraron patrones visuales asociados a ellas, que se dan de forma autónoma y permiten su diferenciación (Ekman, 1992).

Hill (2017) resume tres cualidades universales de las emociones y una cualidad adicional:

Un componente de sentimiento: sensaciones físicas incluyendo cambios químicos en el cerebro.

Componente de pensamiento: apreciaciones "racionales" conscientes o intuitivas. Un componente de acción: reacciones expresivas (como sonrisas o fruncir el ceño) al igual que conductas de afrontamiento (pelea o huida). A veces existe un componente sensorial opcional y sería el cuarto.

Componente sensorial: Vista, sonido, etc., que se inmiscuye y sirve como detonador de la respuesta emocional (pp. 86-87).

Las emociones en su función adaptativa generan respuestas conductuales en los seres humanos, es decir, que se puede afectar la conducta por medio de la emoción. La tabla 2 muestra la relación entre estados emocionales primarios y un resultado conductual. 
Tabla 2. Estados emocionales primarios y resultado conductual

\begin{tabular}{|c|c|c|c|c|c|c|c|}
\hline & \multirow{2}{*}{\multicolumn{6}{|c|}{ Estados emocionales primarios }} \\
\hline & & & & & & & \\
\hline & & Felicidad & Sorpresa & Enojo & Temor & Tristeza & Repugnancia \\
\hline \multirow{5}{*}{ 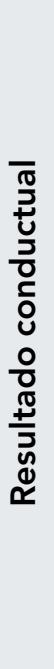 } & $\begin{array}{l}\text { Orientación } \\
\text { a resultados }\end{array}$ & $\begin{array}{l}\text { Elevado } \\
\text { enfoque } \\
\text { en recibir } \\
\text { recompensa }\end{array}$ & $\begin{array}{l}\text { Enfoque } \\
\text { dividido entre } \\
\text { recompensa y } \\
\text { castigo }\end{array}$ & $\begin{array}{l}\text { Elevado } \\
\text { enfoque } \\
\text { en recibir } \\
\text { recompensa }\end{array}$ & $\begin{array}{l}\text { Enfoque } \\
\text { dividido entre } \\
\text { recompensa y } \\
\text { castigo }\end{array}$ & $\begin{array}{l}\text { Elevado } \\
\text { enfoque } \\
\text { en recibir } \\
\text { recompensa }\end{array}$ & $\begin{array}{l}\text { Elevado } \\
\text { enfoque } \\
\text { en evitar el } \\
\text { castigo }\end{array}$ \\
\hline & $\begin{array}{l}\text { Nivel de } \\
\text { atención }\end{array}$ & Alto & Alto & Medio & Medio & Alto & Bajo \\
\hline & $\begin{array}{l}\text { Predisposición } \\
\text { a la acción }\end{array}$ & $\begin{array}{l}\text { Alta: } \\
\text { búsqueda de } \\
\text { resultados }\end{array}$ & $\begin{array}{l}\text { Alta: } \\
\text { búsqueda de } \\
\text { resultados }\end{array}$ & $\begin{array}{l}\text { Alta: impulsa } \\
\text { la represalia }\end{array}$ & $\begin{array}{l}\text { Baja: demora } \\
\text { congelamiento }\end{array}$ & $\begin{array}{l}\text { Baja: demora } \\
\text { congelamiento }\end{array}$ & Alta: evitar \\
\hline & $\begin{array}{l}\text { Tolerancia al } \\
\text { riesgo }\end{array}$ & $\begin{array}{l}\text { Alto riesgo, } \\
\text { baja } \\
\text { recompensa }\end{array}$ & NA & $\begin{array}{l}\text { Alto } \\
\text { riesgo, alta } \\
\text { recompensa }\end{array}$ & $\begin{array}{l}\text { Bajo } \\
\text { riesgo, baja } \\
\text { recompensa }\end{array}$ & $\begin{array}{l}\text { Alto } \\
\text { riesgo, alta } \\
\text { recompensa }\end{array}$ & NA \\
\hline & $\begin{array}{l}\text { Toma de } \\
\text { decisiones }\end{array}$ & $\begin{array}{l}\text { Rápida (sin } \\
\text { preocupación) }\end{array}$ & $\begin{array}{l}\text { Cauta } \\
\text { (incierta) }\end{array}$ & $\begin{array}{l}\text { Rápida } \\
\text { (impulsiva) }\end{array}$ & $\begin{array}{l}\text { Cauta } \\
\text { (preocupación } \\
\text { del resultado) }\end{array}$ & $\begin{array}{l}\text { Cauta } \\
\text { (demora) }\end{array}$ & $\begin{array}{l}\text { Rápida (sin } \\
\text { preocupación) }\end{array}$ \\
\hline
\end{tabular}

Fuente: tomado de Hill (2017 p. 93).

Las relaciones entre los estados emocionales y las conductas muestran que se pueden inhibir conductas que requieran acción en la toma de decisiones. Para el objeto de estudio, un paciente que tenga sentimientos de temor o tristeza evocados de forma inconsciente por estímulos sensoriales genera congelamiento en la toma de decisiones y normalmente animadversión al tratamiento.

\section{Materiales y métodos}

Se propuso un estudio exploratorio con aproximación híbrida (cualitativa y cuantitativa). Para el estudio cualitativo se utilizó la técnica de entrevista a profundidad con exposición a sustancias odoríficas asociadas a las prácticas de salud y odontología (alcohol y eugenol) y como estímulo moderador se utilizó el aroma a talco de bebé para evocar recuerdos positivos. ${ }^{5}$ Para este se definieron dos poblaciones: los sujetos que han tenido experiencias

5 Se puede entender el talco de bebé como un estimulante del sistema límbico ya que normalmente se asocia con ternura, bebés, hijos o infancia (Gomez \& Manci, 2014). 
positivas odontológicas previas y aquellos que manifestaron experiencias odontológicas negativas. La muestra tomada fue de 10 y 7 sujetos respectivamente (tabla 3 ).

Tabla 3. Distribución muestral de las poblaciones de estudio (análisis cualitativo)

\begin{tabular}{|c|c|c|c|c|c|c|c|}
\hline & Sexo & Edad & $\begin{array}{l}\text { Experiencia } \\
\text { odontológica }\end{array}$ & $\begin{array}{l}\text { Experiencia } \\
\text { aroma } \\
\text { talco bebé }\end{array}$ & $\begin{array}{c}\text { Experiencia } \\
\text { aroma a } \\
\text { Eugenol }\end{array}$ & Asociación semántica & $\begin{array}{l}\text { Somatización } \\
\text { de estímulos }\end{array}$ \\
\hline 1 & $\mathrm{~F}$ & 64 & + & + & $\mathrm{R}$ & Muerte Calzas & $\mathrm{Si}$ \\
\hline 2 & $\mathrm{~F}$ & 32 & $\mathrm{R}$ & + & - & Odontología / dolor / miedo & No \\
\hline 3 & M & 39 & - & + & - & Dolor & No \\
\hline 4 & M & 18 & - & + & - & Odontología & No \\
\hline 5 & $\mathrm{~F}$ & 44 & - & + & - & Muerto & Si \\
\hline 6 & M & 73 & + & + & - & No lo reconoce/No le gusta & No \\
\hline 7 & M & 38 & $\mathrm{R}$ & + & - & Odontología & No \\
\hline 8 & $\mathrm{~F}$ & 29 & + & + & + & Odontología & No \\
\hline 9 & M & 33 & $\mathrm{R}$ & - & + & Odontología & No \\
\hline 10 & M & 23 & - & + & - & Odontología & $\mathrm{Si}$ \\
\hline 11 & M & 24 & + & + & + & Odontología & No \\
\hline 12 & M & 28 & + & + & $\mathrm{R}$ & Clavo de olor & No \\
\hline 13 & $\mathrm{~F}$ & 28 & + & + & - & Monte & No \\
\hline 14 & $\mathrm{~F}$ & 31 & + & + & $\mathrm{R}$ & No lo reconoce/No le gusta & No \\
\hline 15 & $\mathrm{~F}$ & 68 & - & + & - & Odontología & No \\
\hline 16 & $\mathrm{~F}$ & 66 & - & + & - & Clavo de olor & No \\
\hline 17 & $\mathrm{~F}$ & 24 & - & + & - & Odontología & No \\
\hline
\end{tabular}

Fuente: elaboración propia.

Para el estudio cuantitativo se utilizaron dos herramientas biométricas: la respuesta galvánica de piel (GSR) y la lectura facial. ${ }^{6}$ Se tomó una muestra de 12 pacientes, de los cuales tres manifestaron haber tenido experiencias odontológicas traumáticas previas. Todos habían sido sometidos al eugenol en sus tratamientos.

\section{Protocolo de intervención}

Los participantes de prueba, elegidos mediante muestreo a juicio, fueron conducidos a la sala de creatividad del laboratorio de mercadeo ubicado en las instalaciones de la Universidad

6 El GSR (Respuesta galvánica de piel, por sus siglas en inglés) es un aparato usado con el fin de registrar la intensidad emocional de las reacciones frente a un estímulo por medio de las activaciones en la conductancia de la piel. El Face Reader es un tipo de software basado en codificación facial que permite la decodificación de movimientos faciales en emociones predeterminadas que se entienden como variables multinomiales. Incluye seis expresiones emocionales: felicidad, tristeza, rabia, sorpresa, miedo y disgusto. 
del Norte, ${ }^{7}$ uno a uno se les explicó el propósito de la prueba. Se les entregó un consentimiento informado el cual fue firmado por cada participante. Se manifestó en el acercamiento, que la prueba era no invasiva sin riesgo para su salud y conducente a la toma de medidas biométricas mediante dispositivos electrónicos mediante la exposición a estímulos.

Para realizar las mediciones de cada participante se realizó el siguiente procedimiento:

Se sentó a la persona investigada en una silla y se procedió a la instalación del equipo GSR y a la calibración del Face Reading. Los equipos fueron programados previamente para dos estímulos. El equipo hace el proceso de calibración de la pupila mediante una imagen fija que permite primero registrar la dilatación máxima, luego la mínima y luego la neutra en espacios de ocho segundos entre cada una. Luego se hace una calibración interna por 100 segundos (estado de latencia), que le permite al investigador dar las instrucciones del experimento. El guion suministrado de viva voz por el investigador fue el siguiente:

Bienvenido, gracias por su participación. El estudio tal y como consta en el consentimiento informado que por usted fue firmado no tiene implicaciones en su salud ni representa ningún riesgo. Lo vamos a someter a dos estímulos odoríferos. Haremos luego de la exposición algunas preguntas y seguiremos al siguiente. Sírvase a estar cómodo (sic) observando el punto de enfoque de la pantalla. Una vez el sistema esté calibrado daremos las instrucciones para comenzar.

El primer estímulo fue el olor a talco de bebé. Se usó la sustancia talco de droguería Juliao que evoca el olor a talco Johnson \& Johnson clásico. ${ }^{8}$ Luego se le hizo la pregunta al participante: “QQué le viene a la mente con este olor?”. Acto seguido al participante se le pide oprimir la tecla de barra espaciadora en el computador y se sigue al siguiente estímulo, el olor a eugenol, y se hace la misma pregunta. Realizado el proceso de medición se retiran los dispositivos, se agradece, se entrega un obsequio y se despide a la persona.

\footnotetext{
Universidad del caribe colombiano ubicada en la ciudad de Barranquilla, Colombia. www.uninorte.edu.co

Droguería Juliao es una botica conocida en la zona norte de Colombia que distribuye y fabrica diversos productos para la salud, la belleza, el bienestar y agroquímicos diversos. Su página web es http://www.drogueriasjuliao.com/
} 


\section{Resultados}

\section{Aproximación cualitativa}

En la figura 1, producto de las entrevistas, se esquematiza las relaciones negativas y positivas de dos estimulos olfativos. En la parte izquierda se aprecia la relación negativa con el Eugenol, con expresiones como "siento mareo, me aprieto y sufro de tos, mejor dicho, me hace un daño en la salud". Se evidencian incluso relaciones psicosomáticas en las narrativas: "cada que percibía este olor me daba dolor de cabeza". Mientras que en la parte derecha de la figura 1 se muestran las relaciones positivas especialmente con talco de bebé tales como "el olor de nacimiento de mis tres hijas que era el olor favorito para mi".

Las personas que no asocian el eugenol con odontología son jóvenes, quienes en cambio lo asocian con comida y clavito. Así como dice el condicionamiento clásico, si no hay experiencias previas, no hay aprendizaje y no se asocia a la odontología.

Toda la muestra que se tuvo en cuenta había sido sometida a tratamientos odontológicos previos. Unos más invasivos que otros. No se pudo determinar con quienes había sido usado el compuesto a base de eugenol. Las edades de la muestra oscilaron entre los $18 \mathrm{y}$ 73 años con 8 hombres y 9 mujeres (tabla 3).

De los entrevistados, siete consideraron que sus experiencias odontológicas habían sido positivas y otros siete manifestaron que habían sido negativas, mientras que tres hablaron de que las experiencias odontológicas habían sido regulares.

En relación con la exposición al aroma de talco de bebé, se encontraron 16 buenas experiencias al enfrentar el estímulo y solo una experiencia reportada como negativa. Las manifestaciones semánticas más comunes ante la exposición a talco de bebé fueron las expresiones de ternura, bebés, nacimiento y cuidado.

Tres de los participantes manifestaron sentir malestares físicos como náuseas, vómitos, rinitis y cefaleas al experimentar exposición a olores fuertes. Casualmente, a ninguno de ellos les agradó el olor a eugenol por considerarlo muy penetrante. 
Once de los participantes asociaron el olor a eugenol directamente con la práctica odontológica, tres más con el olor a clavo de olor y otros tres no lo asociaron con nada, estos últimos pertenecen al grupo de los que tuvieron experiencias positivas durante los tratamientos odontológicos. Otro tres de los participantes asociaron el olor a eugenol con muerte.

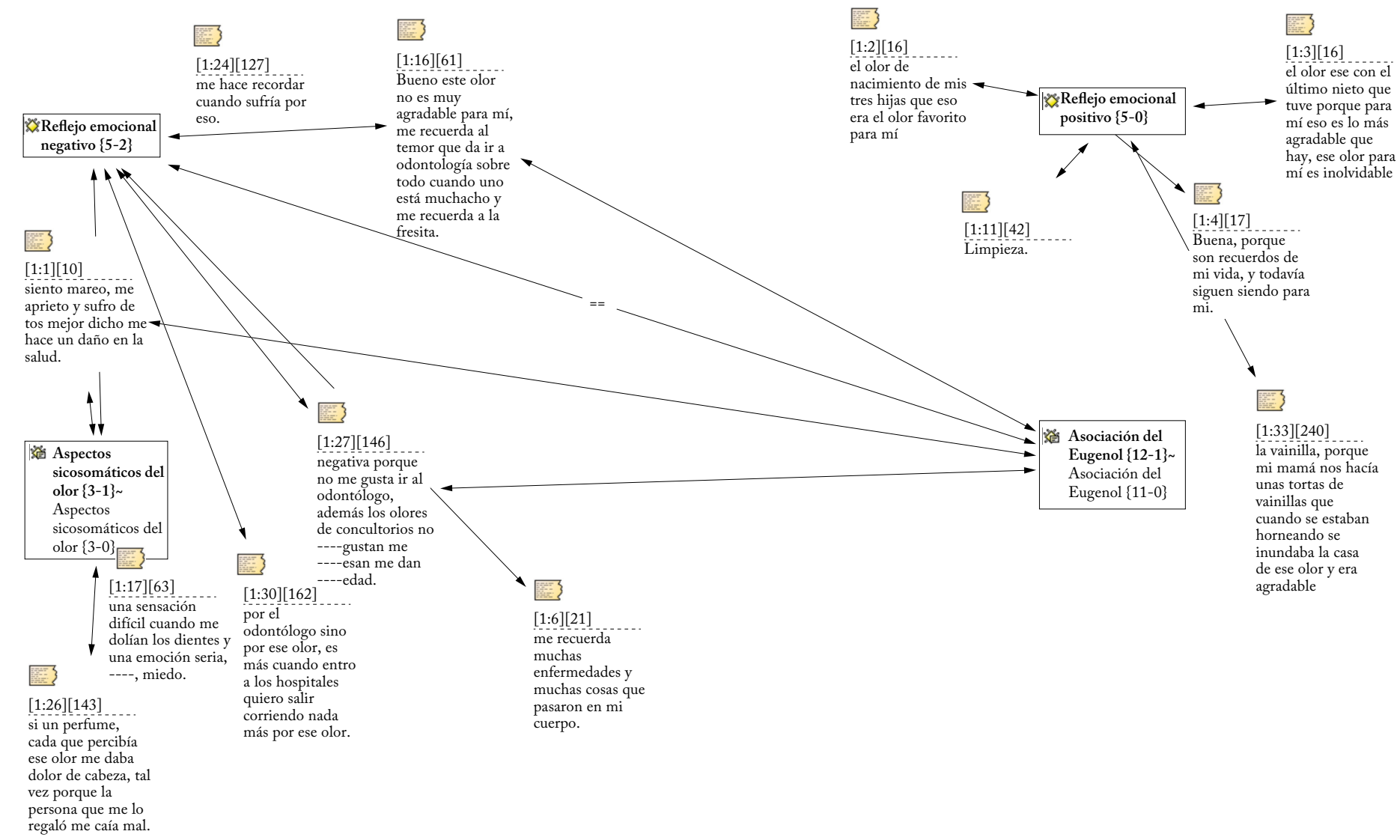

Figura 1. Asociaciones al estímulo olfativo: eugenol - talco de bebé

\section{Aproximación cuantitativa: Descripción de cambios fisiológicos y emocionales de los participantes del estudio a través de biometría con el estímulo eugenol}

En la figura 2 se aprecia a los participantes cuando son conectados a los aparatos biométricos siguiendo el protocolo descrito en el aparte materiales y métdos. En la tabla 4 se hace un resumen de la información y los datos de los participantes. 
Tabla 4. Descripción de la muestra para análisis biométrico

\begin{tabular}{|c|c|c|c|c|c|c|}
\hline & \multirow[b]{2}{*}{ Nombre } & \multicolumn{2}{|c|}{ Sexo } & \multirow[t]{2}{*}{ Edad } & \multicolumn{2}{|c|}{$\begin{array}{c}\text { ¿Considera que su } \\
\text { tratamiento odontológico } \\
\text { fue traumático? }\end{array}$} \\
\hline & & Hombre & Mujer & & Sí & No \\
\hline 1 & Participante 1 & & $x$ & 47 & & $x$ \\
\hline 2 & Participante 2 & & $x$ & 31 & & $x$ \\
\hline 3 & Participante 3 & & $x$ & 28 & $x$ & \\
\hline 4 & Participante 4 & & $x$ & 31 & & $x$ \\
\hline 5 & Participante 5 & & $x$ & 32 & $x$ & \\
\hline 6 & Participante 6 & $x$ & & 34 & $x$ & \\
\hline 7 & Participante 7 & & $x$ & 27 & & $x$ \\
\hline 8 & Participante 8 & $x$ & & 17 & & $x$ \\
\hline 9 & Participante 9 & & $x$ & 40 & $x$ & \\
\hline 10 & Participante 10 & & $x$ & 34 & & $x$ \\
\hline 11 & Participante 11 & & $x$ & 22 & & $x$ \\
\hline 12 & Participante 12 & & $x$ & 28 & & $x$ \\
\hline
\end{tabular}

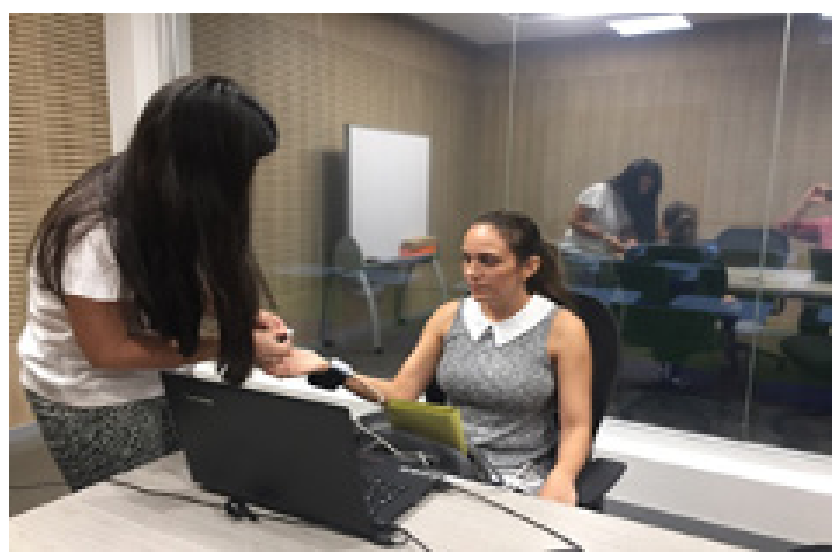

Figura 2. Abordaje biométrico

El modelo de aproximación a las métricas emocionales se basa en el modelo circumplejo afectivo (Rusell, 1980), que como modelo dimensional entiende las transiciones afectivas como estados correlacionados y continuos (Posner, Rusell \& Peterson, 2005). Rusell identifica la emoción con características de dirección e intensidad. El modelo lo representa por medio del eje de valencia (abscisas) que representa la dirección (si es positiva-agradable o negativa-desagradable) y el eje de nivel de intensidad o exaltación emocional —arousal_, que representa la intensidad o grado de activación neuronal (figura 3). 


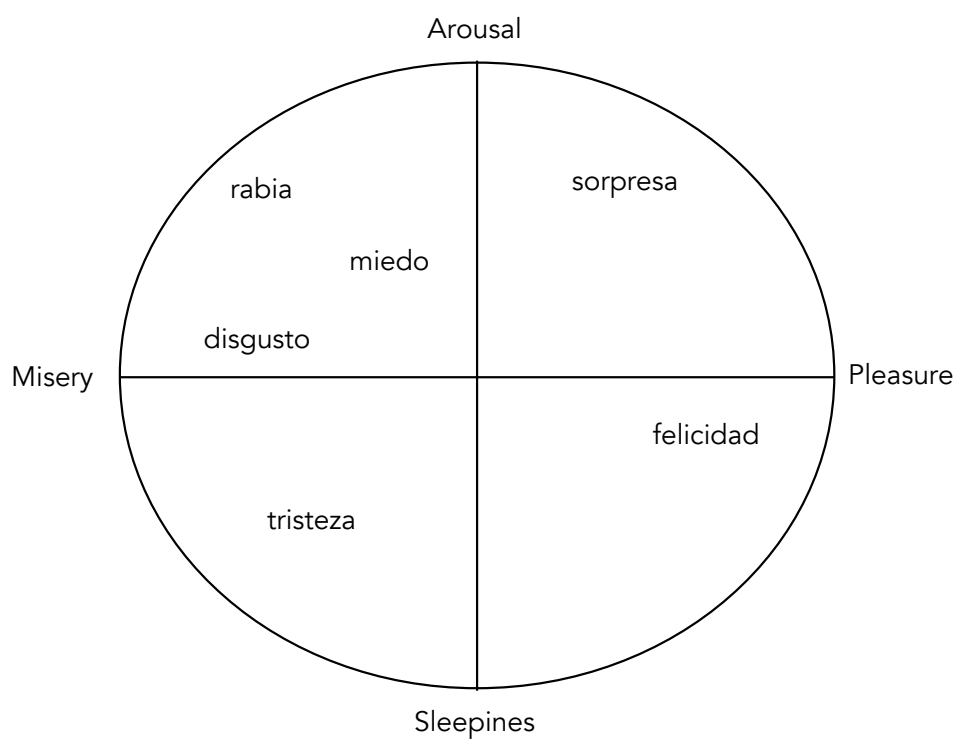

Figura 3. Modelo circumplejo afectivo de Rusell

Fuente: Rusell (1980, p. 1164) modificado por el autor a partir de las emociones registradas en el software usado.

Teniendo en cuenta el software usado los tipos de emociones registrados por este son seis: felicidad, tristeza, rabia, sorpresa, miedo y disgusto. Las variables de respuesta de los aparatos biométricos utilizados fueron arousal y valencia (Lang, 1995).

Arousal. Nivel de intensidad o excitación emocional: refleja la energía que se da o invierte durante la emoción, se traduce como la respuesta del sistema simpático que se genera en la emoción.

Valencia: "dimensión principal sobre la cual se construye la experiencia emocional, es el componente motivacional de la emoción (placer vs. displacer)" (Gantiva \& Camacho, 2016, p. 56). 


\section{Abordaje biométrico}

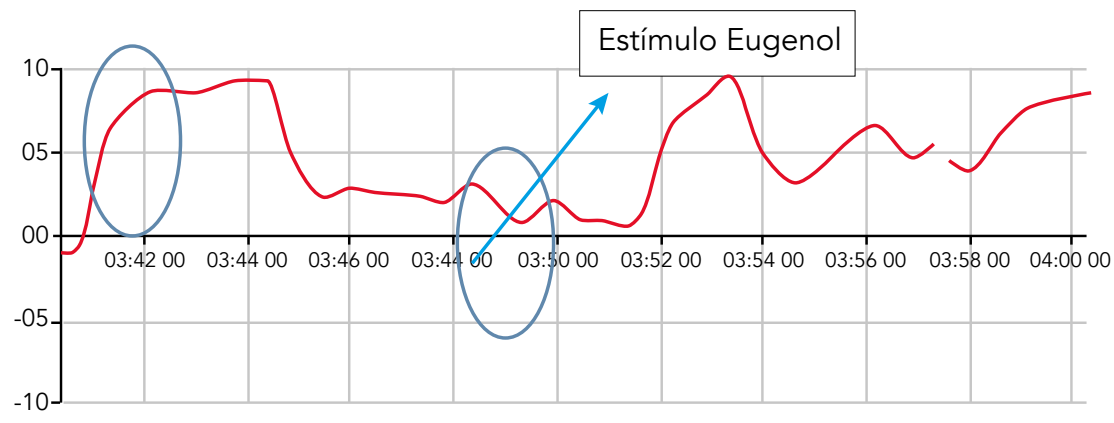

Valance

Figura 4. Valencia sujeto 1

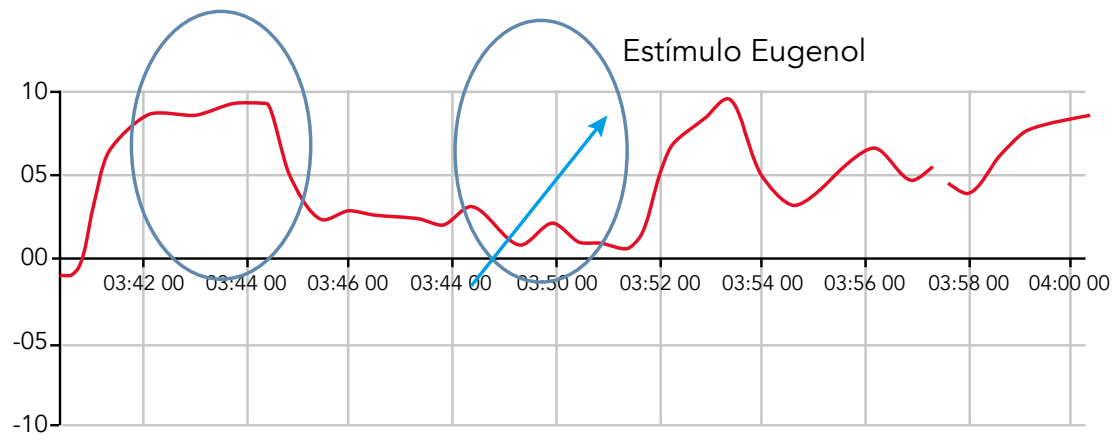

Valance

Figura 5. Valencia sujeto 2

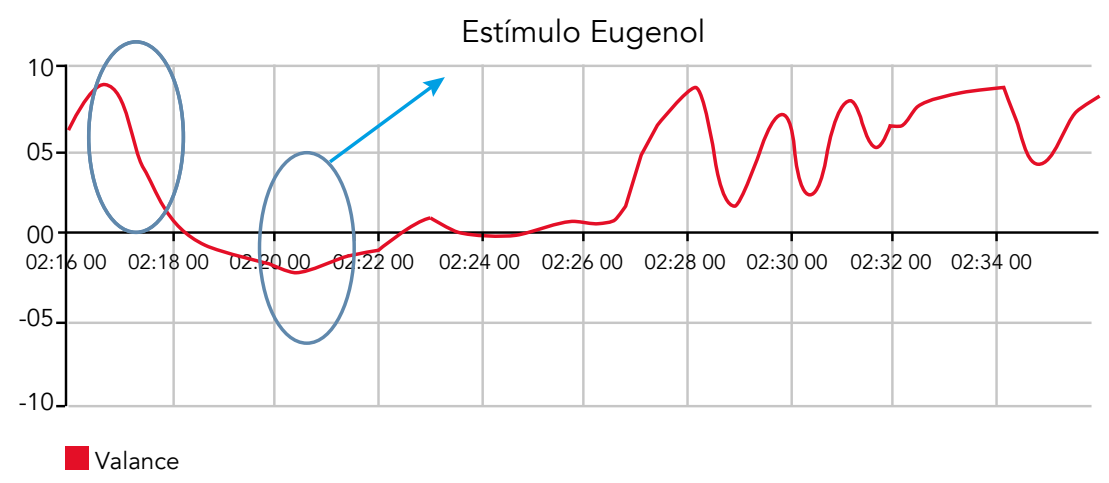

Figura 6. Valencia sujeto 3 


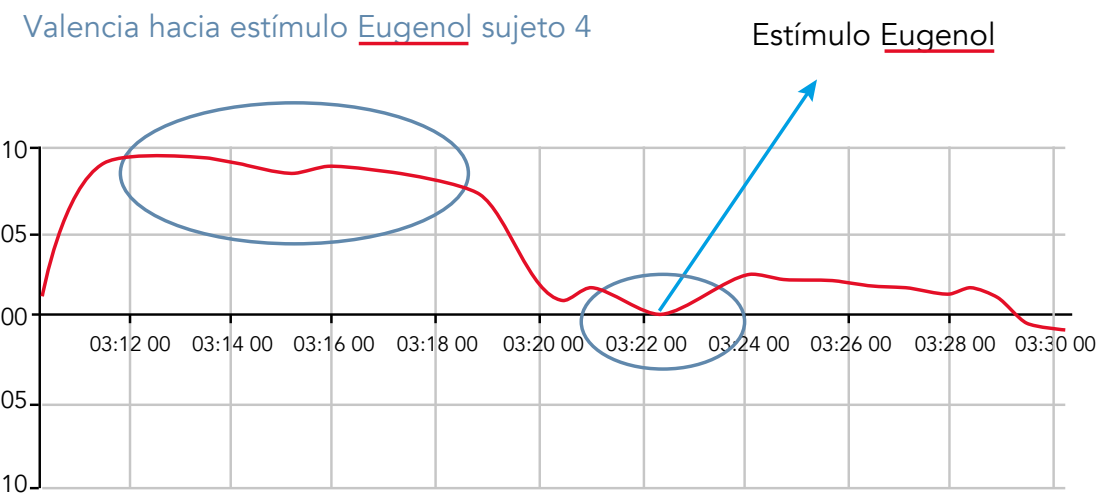

Valance

Figura 7. Valencia sujeto 4

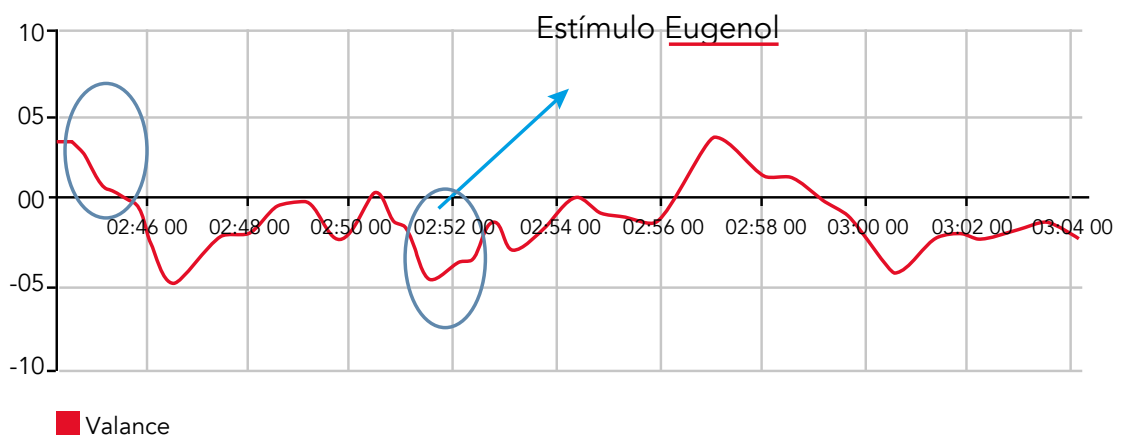

Figura 8. Valencia sujeto 5

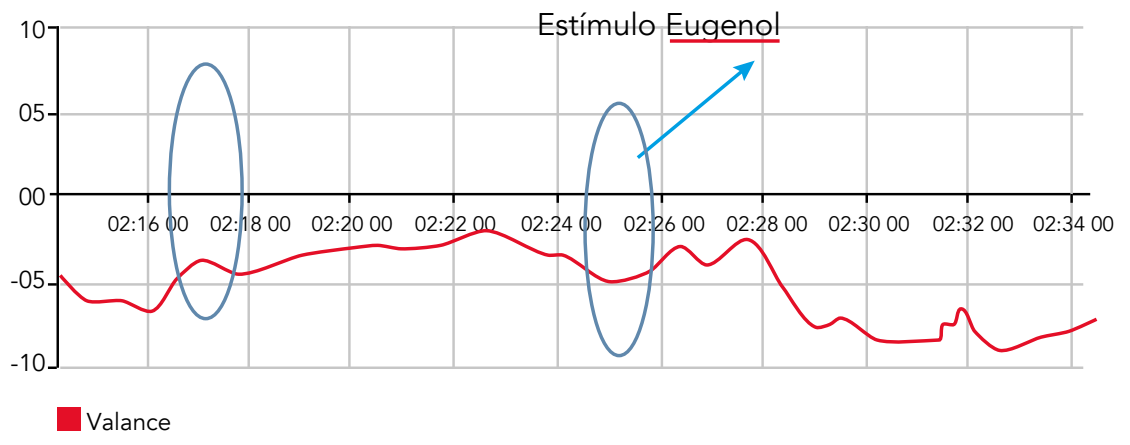

Figura 9. Valencia sujeto 6 


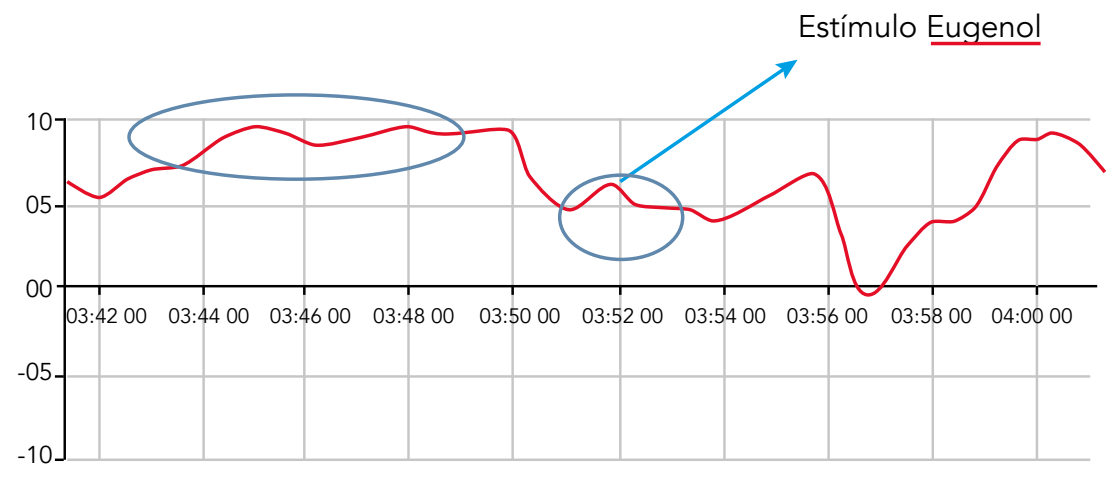

Valance

Figura 10. Valencia sujeto 7

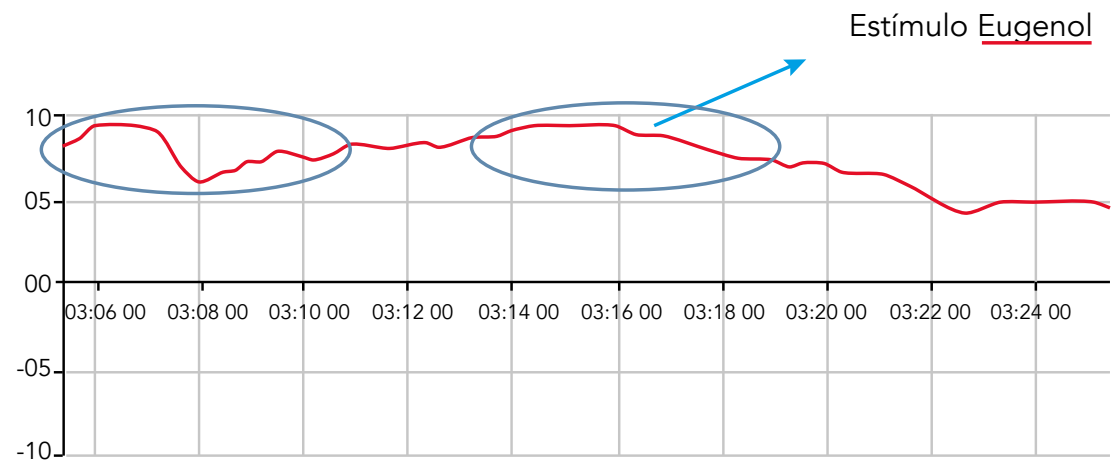

Valance

Figura 11. Valencia sujeto 8

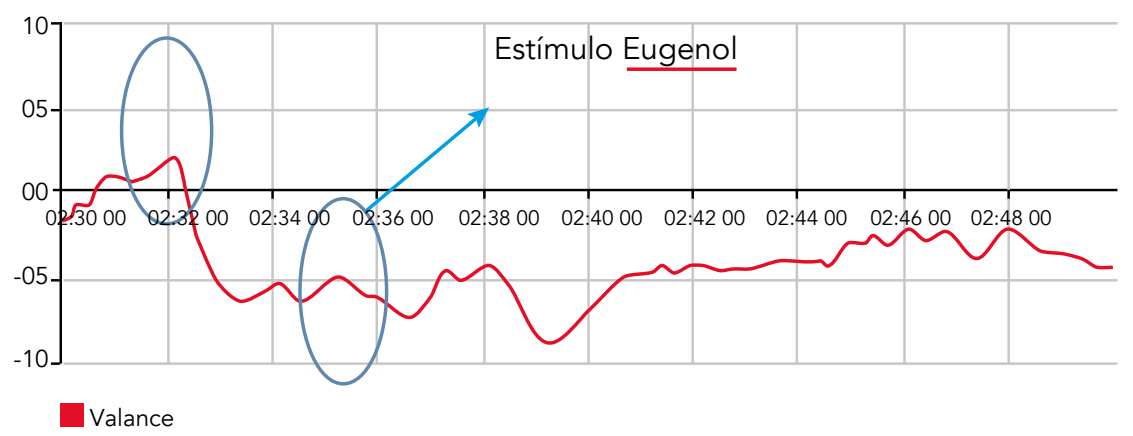

Figura 12. Valencia sujeto 9 


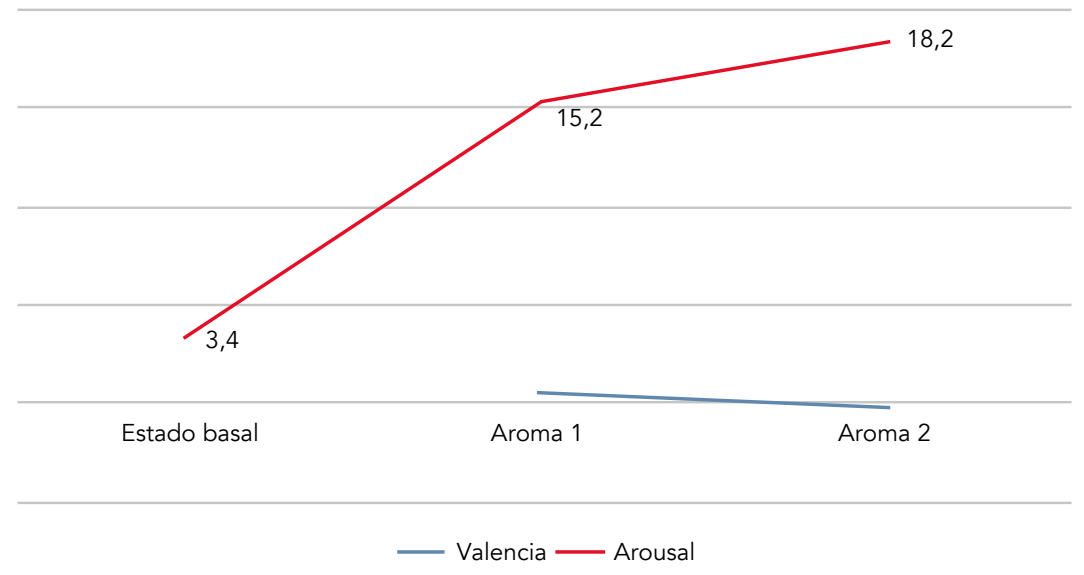

Figura 13. Nivel de activación y nivel de valencia asociada

Como se puede observar, las métricas ofrecidas entre arousal y valencia son variables de estado (fácilmente verificables de forma externa) sin influencia del fenómeno de respuesta revelada (asumir como cierta la respuesta del entrevistado). Sus métricas tienen en cuenta las reacciones fisiológicas del estímulo. Para el caso del nivel de activación (arousal) se verifica el incremento tónico de la activación electrodérmica, medida a través del GSR. En el caso de las emociones, la medición se realiza a través del software Noldus Face Reader ${ }^{\mathrm{TM}}$, que tiene un algoritmo que permite la devolución de seis emociones percibidas mediante tres pasos: reconocimiento de expresión facial mediante el algoritmo (Viola \& Jones, 2001) y entrega de estas como variables multinomiales.

Se observa en el gráfico acumulado (figura 13) que existe un nivel de activación positivo marcado para el estímulo olfativo de talco de bebé con una valencia positiva moderada de 0,55 (ubicada en el cuadrante de placer en el modelo Rusell). Esta puede interpretarse en términos de felicidad. Así mismo, se observa en el estímulo olfativo eugenol un nivel de activación mayor con valencia negativa de baja intensidad -0,11, la cual puede interpretarse como tristeza.

\section{Discusión}

La aproximación de estudios que involucran los estímulos de aromas y su influencia en el mercadeo han sido estudiados desde diferentes perspectivas y normalmente se asocian a propósitos comerciales, conocido como scent marketing (Emsenhuber, 2009). Involucrar 
el ámbito de la salud al análisis de este estímulo en la práctica permite el entendimiento de las influencias de los odotipos en la respuesta emocional del paciente en procura de extenderle condiciones que le permitan una mejor experiencia. Este fenómeno no solo tendría en la práctica resultados más asertivos para el odontólogo tratante, sino también efectos positivos en la adherencia al tratamiento. Se pudo verificar que existe un cambio de respuesta fisiológica evidenciable con los estímulos olfativos y que es posible aproximarse a correlatos entre la experiencia previa asociada a la variable tratamiento y su respuesta. El abordaje cualitativo permitió encontrar patrones claros entre traumas y aromas asociados que disparaban reacciones somáticas. Por su parte, los niveles de activación (aurosal) detectados por el software encuentran niveles de detección claros en dos estímulos olfativos. El primero relacionado con el talco de bebé y el segundo con el eugenol.

De las nueve mediciones realizadas, se pudo observar que los sujetos 3, 5, 6 y 9 (ver figuras 6, 8, 9 y 12 respectivamente) mantuvieron una valencia negativa en el estímulo del eugenol con un nivel de activación medio. No es de extrañar que estos individuos contestaran afirmativamente la pregunta “ ¿Considera que su tratamiento odontológico fue traumático?". Por su parte, los sujetos 1, 2, 4, 7 y 8 (ver figuras 4, 5, 7, 10 y 11 respectivamente) mantuvieron niveles de valencia positivos con niveles de activación medios, la respuesta a la pregunta en este caso fue negativa.

\section{Conclusiones}

Se pudo describir la respuesta emocional a un estímulo olfativo de un paciente odontológico mediante el uso de técnicas biométricas. Así mismo, se pudieron explorar relaciones similares mediante aproximaciones cualitativas que hicieron convergencia en términos de respuestas conductuales asociadas al condicionamiento clásico.

Comprender esto en términos de las variables de control de mercadeo significa la posibilidad de intervenir los estímulos asociados negativamente, en procura de una primera sensación organoléptica que minimice las activaciones somáticas iniciales desprendidas 
del estímulo. La intervención en este aspecto puede contribuir a una mejor adaptación del paciente y con ella a un aumento en la probabilidad de retención por parte del odontólogo.

El artículo se convierte en un punto de partida para otras aproximaciones metodológicas entre las que se sugieren abordajes experimentales con una muestra más grande.

\section{Referencias}

Avendaño Castro, W. R., Paz Montes, L. S., \& Rueda Vera, G. (2015). Estímulos auditivos en prácticas de neuromarketing. Caso: Centro Comercial Unicentro, Cúcuta, Colombia. Cuadernos de Administración, 31(53).

Brakus, J. J., Schmitt, B. H., \& Zarantonello, L. (2009). Brand experience: what is it? How is it measured? Does it affect loyalty? Journal of marketing, 73(3), 52-68.

Bruce Goldstein, E. (2005). Sensación y percepción. Madrid. International Thomson cop2006 Ayala, F, 30-18.

Carballo, J. (1997). Clasificación de trastornos de ansiedad en el paciente odontológico: una propuesta. Revista ADM, 54(4), 207.

Caycedo, C., Cortés, O. F., Gama, R., Rodríguez, H., Colorado, P., Caycedo, M., ... Palencia, R. (2008). Ansiedad al tratamiento odontológico: características y diferencias de género. Suma Psicológica, 15(1), 259-278. https://doi.org/10.14349/sumapsi2008.26

Diener, E. (1999). Introduction to the special section on the structure of emotion. Journal of personality and Social Psychology, 76(5), 803.

Ekman, P. (1992). An argument for basic emotions. Cognition E emotion, 6(3-4), 169-200.

Frow, P., \& Payne, A. (2007). Towards the 'perfect'customer experience. Journal of Brand Management, 15(2), 89-101.

Gantiva, C., \& Camacho, K. (2016). Características de la respuesta emocional generada por las palabras: un estudio experimental desde la emoción y la motivación. Psychologia. Avances de la disciplina, 10(2), 55-62.

Gentile, C., Spiller, N., \& Noci, G. (2007). How to sustain the customer experience: An overview of experience components that co-create value with the customer. European management journal, 25(5), 395-410.

Gómez Ramírez, C., Manzi Puertas, M. A., \& Galindo Becerra, T. (2014). El scent marketing: una revisión bibliográfica. Revista científica: Pensamiento y Gestión. 
Hall, J. E. (2011). Guyton y Hall. Tratado de fisiología médica. Elsevier Health Sciences.

Hill, D. (2017). Emotionomics: El poder de las emociones para el éxito de los negocios. Grupo Editorial Patria.

Holbrook B. M. and Hirshman C. E. (1982). "The Experiential Aspects of Consumption: Consumer Fantasies, Feeling and Fun”, Journal of Consumer Research. Vol.9, September, pp.132-140.

Kemper, T. (1987). How many emotions are there? Wedding the social and the autonomic components. American journal of Sociology, 93(2), 263-289.

Lizardi, P. G., \& Jiménez, H. A. G. (2012). Ansiedad en el paciente odontológico. Urgencias médicas en odontología, 36.

Ming-Shing, L, Huey-Dar, H, \& Ming-Fen, Y. (2010). The study of the relationships among experiential marketing, service quality, customer satisfaction and customer loyalty. International Journal of Organizational Innovation, 3(2).

Moral, M., \& Fernández, M. (2012). Nuevas tendencias del marketing: el marketing experiencial. Revista Entelequia, 14, 237.

Morales García, Higinio, M., \& Priego-Álvarez, H. (2008). La importancia del marketing odontológico en el ejercicio profesional. Horizonte Sanitario, 7(2).

Mukiira, E. M., Musau, M. C., \& Munyao, M. J. (2017). Effect of experiential marketing in building brand equity: A case of selected Unilever Tanzania brands. International Journal of Supply Chain Management, 2(1), 1-31.

Páez, D., Ruiz, J., Gailly, O., Kornblit, A., Wiesenfeld, E., \& Vidal, C. (1997). Clima emocional: su concepto y medición mediante una investigación transcultural. Revista de Psicología Social, 12(1), 79-98.

Pine, B. J., Pine, J., \& Gilmore, J. H. (1999). The experience economy: Work is theatre \& every business a stage. Harvard Business Press.

Posner, J., Russell, J. A., \& Peterson, B. S. (2005). The circumplex model of affect: An integrative approach to affective neuroscience, cognitive development, and psychopathology. Development and Psychopathology, 17(3), 715-734.

Rozo, J. A., \& Pérez-Acosta, A. M. (2015). Condicionamiento clásico y cognición implícita. Acta Colombiana de Psicología, 9(1), 63-75.

Russell, J. 1980. A Circumplex Model of Affect. Journal of Personality \& Social Psychology, $39(6), 1161-78$.

Schmitt, B.H. (1999) Experiential marketing: How to get customers to sense, feel, think, act and relate to your company and brands. New York, The Free Press. 
Till, B. D., \& Lynn Priluck, R. (2000). Stimulus generalization in classical conditioning: An initial investigation and extension. Psychology \& Marketing, 17, 55-72.

Vinaccia, S., Bedoya, L. M., \& Valencia, M. (1998). Odontología y psicología. Disminución de la ansiedad en pacientes sometidos a cirugía odontológica. Revista Latinoamericana de Psicología, 30(1), 67-82.

Viola, P., \& Jones, M. (2001, December). Rapid object detection using a boosted cascade of simple features. https://www.cs.cmu.edu/ efros/courses/LBMV07/Papers/viola-cvpr-01.pdf 